\title{
Performance Oriented Control over Networks - Switching Controllers and Switched Time Delay -
}

\author{
Sandra Hirche*, Chih-Chung Chen and Martin Buss
}

\begin{abstract}
The stability and performance of a networked control system (NCS) strongly depends on the communication quality, e.g. of the communication time delay. Aiming at performance oriented control over networks in the presence of piecewise constant time delay, two novel control approaches are investigated. In the first approach, the time delay is monitored and an appropriate controller is selected. The second approach is based on the Quality-of-Service communication concept, where the time delay is adjustable and related to the network cost. Aiming at an optimal trade-off between network cost and control performance the controller together with the time delay is switched. Both approaches result in a switched system with switched (piecewise constant) delays. Sufficient stability conditions for the resulting switched time delay system are presented using a piecewise continuous Lyapunov-Razumikhin function. A common Lyapunov function is derived for linear time-invariant symmetric subsystems. The performance benefits for both approaches are demonstrated in numerical examples.
\end{abstract}

\section{INTRODUCTION}

In the view of affordability, widespread usage and well developed infrastructure, communication networks are very attractive for the signal transmission in control systems. This comes, however, at the cost of a no longer ideal signal transmission. Especially, time delay associated with the data transmission has a strong influence on the stability and the performance of the closed loop system, see [1], [2] for a general overview on control challenges and approaches for networked control systems (NCS). In the current NCS literature the communication quality is assumed to be given in advance, accordingly stabilizing controllers are designed. In this paper two alternative approaches dealing with the time delay problem are proposed. In the first approach with time delay dependent controller switching, the time delay is monitored and an appropriate controller is selected online to ensure good performance. The time delay is assumed to be piecewise constant. This assumption is reasonable, e.g. in Token ring networks, where by the removal or the addition of a network node the otherwise constant time delay decreases/increases in a discontinuous manner, further also for route switching in multi-route networks.

In the second approach, the Quality-of-Service (QoS) control approach, the communication time delay is considered to be adjustable in realtime. This is motivated by the QoS concept from the networking community, which refers to the

\footnotetext{
Institute of Automatic Control Engineering, Technische Universität München, D-80290 München, Germany; http://www.lsr.ei.tum.de, chendlsr.ei.tum.de, m.buss@ieee.org

* Visiting researcher at the Fujita Lab., Dept. of Mechanical and Control Engineering, Tokyo Institute of Technology, 152-8552 Tokyo, Japan s.hirchedieee.org
}

capability of a network to provide different communication quality to different network traffic classes. Guaranteed low time delay results in very good control performance, but requires the provision of large network resources, i.e. induces high network cost. Aiming at a trade-off between control performance and network cost it is proposed to switch the time delay together with an appropriate controller. The time delay is assumed piecewise constant.

As a result, in both considered approaches the time delay as well as the controller are switched resulting in a switched system with switched time delay. Switched (hybrid) systems are dynamical systems that consist a set of finite subsystems and a logical rule that orchestrates the switchings between them. The stability of the subsystems themselves is not sufficient for the stability of the overall system [3], [4]. Stability with arbitrary switching can be ensured by a common Lyapunov function which is difficult to construct in general. The concept of piecewise continuous Lyapunov functions is developed in [5]-[7]. Alternatively, dwell-time based switching is considered in [8], [9]. All these approaches, however, do not consider time delay. Most prominent approaches in the rich literature on time delay systems, see e.g. [10][12] and references therein, are Lyapunov-like approaches based either on the Razumikhin or the Krasovskii method. Switched time delay systems are considered in [13], [14], where the common Lyapunov function approach is extended and in [15] adopting a dwell-time approach. However, a constant time delay is considered there. To the best knowledge of the authors there are no stability and performance results available for switched systems with switched time delays. Main contribution of this paper is a sufficient condition for the stability of switched systems with switched (piecewise constant) delays based on a piecewise continuous Lyapunov function approach and a common Lyapunov approach for symmetric systems. The performance of both considered approaches are studied in numerical examples. Benefits in terms of improved control performance are shown for the delay-dependent controller switching approach. An optimal trade-off between control performance and network cost is achieved for the QoS control approach using a hybrid optimal control algorithm from [16].

The remainder of the paper is organized as follows: The problem definition along with preliminaries on the stability of switched systems and time delay systems are given in Section II. In Section III, the main result on stability of switched systems with switched time delay is presented, followed by application examples with performance considerations in Section IV. 


\section{Problem Formulation And Preliminaries}

A networked control system with synchronously switching controllers and delays is considered in this paper; the delays are considered piecewise constant. Comprising hybrid dynamics as well as delay system dynamics it can be described by a switched delay differential functional of the form

$$
\dot{x}=f_{\sigma}\left(x_{t}\right), \quad x_{t_{0}}=\phi .
$$

where $\sigma:[0, \infty) \rightarrow \mathcal{I}$ is a piecewise continuous switching signal takes value on the finite set $\mathcal{I}:=\{1, \ldots, N\}$, $x_{t}=x(t+\theta), \theta \in\left[-\tau_{\sigma}, 0\right]$ indicates the dependence of the state derivative on the past states, $x \in \mathbb{R}^{n}$, and $\tau_{\sigma} \in \mathbb{R}^{+}$represents the piecewise constant time delay. The initial condition is given by the continuous trajectory $x_{t_{0}}=\phi=x\left(t_{0}+\theta\right), \theta \in\left[-\tau_{\sigma\left(t_{0}\right)}, 0\right]$. Note that without switching, i.e. $\sigma(t)$ constant, the classical delay differential functional modeling time delay systems [12] is recovered. With zero time delay $\tau_{i}=0$ for all $i \in \mathcal{I}$, (1) represents the switched system model in the notation of [17]. It is assumed that there is no jump in the state (state reset) at the switching instants, the solution $x(t)$ is continuous everywhere. The time between any two switches is assumed larger than zero, i.e. there is a finite number of switches in finite time. Later in this paper we consider switched systems where each subsystem in (1) is represented by a linear time-invariant (LTI) time delay system of the form

$$
\dot{x}(t)=A_{i 0} x(t)+A_{i 1} x\left(t-\tau_{i}\right), \quad i \in \mathcal{I},
$$

where $A_{i 0}, A_{i 1} \in \mathbb{R}^{n \times n}$.

In contrast to conventional switched systems, the proposed control structure comprises switching of controllers and switching time delays. An analysis method for such systems has not been published so far.

\section{A. Stability of Time Delay Systems}

Most prominent approaches for the stability analysis of time delay systems are the Lyapunov-Krasovskii approach and the Lyapunov-Razumikhin approach. The Krasovskii approach may provide a necessary and sufficient stability condition, the Krasovskii functional includes the retarded states, its computation is difficult. The Razumikhin approach, see e.g. [12], circumvents the difficulties by considering the Lyapunov function $V(x(t))$ depending on the present state $x(t)$ only. Asymptotic stability is guaranteed if the Lyapunov function $\xi V(x(t)), \xi>1$, does not exceed

$$
\bar{V}\left(x_{t}\right)=\sup _{\theta \in[-\tau, 0]} V(x(t+\theta)),
$$

i.e. the largest value of the Lyapunov function over the past time delay interval $[-\tau, 0][11]$. The time argument is dropped in the following if non-ambiguous. Assuming a quadratic Lyapunov function $V(x)=x^{T} P x$ the delaydependent stability condition can be expressed as LMI. Consider therefore the LTI time delay system (2) without switching.
Corollary 1: [12] The LTI time delay system (2) is asymptotically stable if there exists a real symmetric matrix $P>0$ and real scalars $\alpha>0, \alpha_{1}>0$ such that

$$
\left(\begin{array}{ccc}
M & -P A_{1} A_{0} & -P A_{1}^{2} \\
-A_{1}^{T} A_{0}^{T} P & -\alpha P & 0 \\
-\left(A_{1}^{2}\right)^{T} P & 0 & -\alpha_{1} P
\end{array}\right)<0,
$$

where $M=\frac{1}{\tau}\left[P\left(A_{1}+A_{0}\right)+\left(A_{1}+A_{0}\right)^{T} P\right]+\left(\alpha+\alpha_{1}\right) P$. Proof: See [12].

\section{B. Stability of Switched Systems}

It is well known that switching between stable subsystems may result in instability of the switched system [3], [4]. Let $V_{i}$ denote the Lyapunov function associated with subsystem $i$. An important method for the stability analysis of switched systems is based on the construction of a common Lyapunov function $V(x)=V_{i}(x)$ for all $i \in \mathcal{I}$. Its existence guarantees stability for arbitrary switching sequences. However, a common Lyapunov function is generally difficult to find, typically they are found analytically only for certain system classes, such as linear systems with commuting $A$ matrices [18].

An alternative method to analyze the stability of switched systems is by a piecewise continuous Lyapunov function [5][7]. A (piecewise) continuous Lyapunov function $V_{i}$ is associated to each subsystem $i$, where in general $V_{i}(x) \neq V_{j}(x)$ holds if $i \neq j$. While the Lyapunov function $V_{i}$ decreases when $i$ th subsystem is active, it could however increase when subsystem $i$ is inactive. The concept of piecewise continuous function is to restrict $V_{i}$ at any two consecutive instants $t_{k}>t_{j}$ with $\sigma\left(t_{j}\right)=\sigma\left(t_{k}\right)=i$, and $\sigma\left(t_{s}\right) \neq i$ for $t_{j}<t_{s}<t_{k}$

$$
V_{i}\left(x\left(t_{k}\right)\right)-V_{i}\left(x\left(t_{j}\right)\right) \leq-h\left(\left\|x\left(t_{j}\right)\right\|\right), \forall i \in \mathcal{I},
$$

where $h(\cdot)$ is a positive continuous function with $h(0)=0$, i.e. asymptotical stability of the switched systems is guaranteed if the sequence of Lyapunov function values at the switching instants for each subsystem decreases.

\section{Stability of Switched Time Delay Systems}

In this section sufficient conditions for the stability of switched systems with switching time delay are presented. The concept of piecewise continuous Lyapunov functions is extended, a common Lyapunov function is derived for the special class of symmetric systems.

\section{A. Piecewise Continuous Lyapunov Function by Razumikhin Approach}

Consider the switched time delay system given by (1). The following theorem extends the classical Razumikhin theorem [11] to switched systems with piecewise continuous time delays stating a sufficient condition for stability. Therefore define $u_{1}, u_{2}, w, p$, and $h_{i}$ as continuous nondecreasing positive functions $u_{1}(s), u_{2}(s), w(s), p(s), h_{i}(s)>0$ for $\quad s>0 \quad$ with $\quad u_{1}(0)=u_{2}(0)=h_{i}(0)=0$, $u_{2} \quad$ strictly increasing, $\quad \lim _{s \rightarrow \infty} u_{1}(s)=\infty$. By 
$\dot{V}_{i}(x(t))=\lim _{\Delta \rightarrow 0, \Delta>0} \frac{V_{i}(x(t+\Delta))-V_{i}(x(t))}{\Delta}$ right-hand derivative of $\stackrel{\Delta}{i}_{i}(x(t))$ is denoted, and $\left\|x_{t}\right\|=\sup _{\theta \in\left[-\tau_{\sigma(t)}, 0\right]}\|x(t+\theta)\|$.

Theorem 1: The switched time delay system (1) is globally uniformly asymptotically stable if there exists a continuously differentiable positive definite function $V_{i}$ for each subsystem $i \in \mathcal{I}$ such that for any initial condition $x_{t_{0}}=\phi$ and any $t_{0}$

(i) $V_{i}(0)=0, V_{i}(x)>0$ for $x \in \mathbb{R}^{n} \backslash\{0\}$,

(ii) $u_{1}(\|x\|) \leq V_{i}(x) \leq u_{2}(\|x\|)$,

(iii) $\dot{V}_{i}(x) \leq-w(\|x\|)$ if $V_{i}(x(t+\theta)) \leq p\left(V_{i}(x(t))\right)$ for the time interval where subsystem $i$ is active $t \in\left[t_{j}, t_{j+1}\right), \theta \in\left[-\tau_{i}, 0\right]$,

(iv) $\bar{V}_{i}\left(x_{t_{j+1}}\right)-\bar{V}_{i}\left(x_{t_{j}}\right) \leq-h_{i}\left(\left\|x_{t_{j}}\right\|\right)$ for all $i \in \mathcal{I}$ and all consecutive switching instants $t_{j+1}>t_{j}$ for which $\sigma\left(t_{j+1}\right)=\sigma\left(t_{j}\right)=i$.

Sketch of Proof: First note, that conditions (i)-(iii) represent the classical Razumikhin conditions: Without switching $t_{j}=t_{0}, t_{j+1} \rightarrow \infty$, each subsystem $i \in\{1,2, \ldots, N\}$ is globally uniformly asymptotically stable [12]. Without loss of generality assume the switched time delay system is initialized with subsystem $i=1$ at $t_{0}$. For any given $\varepsilon_{1}>0$, let $0<\delta_{0}<u_{2}^{-1}\left(u_{1}\left(\varepsilon_{0}\right)\right)$. Then for any $t_{0}$ and any initial condition $\left\|x_{t_{0}}\right\|<\delta_{0}$ the state remains bounded $\left\|x_{t}\right\|<\varepsilon_{0}$ for $t>t_{0}$, see [12] for more details. Assume, that at the time instant $t_{2}$ the execution enters subsystem 2. As there is no jump in the states at the switching instant by assumption, the initial condition for subsystem 2 is given by $x_{t_{1}}=x\left(t_{1}+\theta\right)$ with $\theta \in\left[-\tau_{2}, 0\right]^{1}$. Using the same arguments as above, with $\left\|x_{t_{1}}\right\|<\varepsilon_{0}$ there is a $\varepsilon_{1}$ such that $\varepsilon_{0}<u_{2}^{-1}\left(u_{1}\left(\varepsilon_{1}\right)\right)$ such that $\left\|x_{t}\right\|<\varepsilon_{1}$ for $t>t_{1}$. Consider $n \leq N$ consecutive switchings with distinct values of $\sigma$. With the same arguments as above for all possible permutations and for any initial condition $\left\|x_{t_{0}}\right\|<\delta$, for all $t_{0}$, there exist a $\varepsilon>0$ such that $\left\|x_{t_{n}}\right\| \leq \varepsilon$. In the case that any subsystem $i$ becomes re-activated, condition (iv) guarantees that the state trajectory stays within the $\varepsilon$-ball. The switched system is uniformly stable.

For asymptotic stability, observe that there exists a subsystem with an associated infinite sequence of switching times $t_{i, 1}, t_{i, 2}, \cdots$ such that $\sigma\left(t_{i, 1}\right)=\sigma\left(t_{i, 2}\right)=\ldots=i \in \mathcal{I}$ (excluding the trivial case of finite switchings). Due to (iv) the measure $\bar{V}_{i}\left(x\left(t_{i, 1}\right)\right), \bar{V}_{i}\left(x\left(t_{i, 2}\right)\right), \ldots$ is decreasing and positive, there exists a $z \geq 0$ such that $z=\lim _{j \rightarrow \infty} \bar{V}_{i}\left(x\left(t_{i, j}\right)\right)$. As a result for any two consecutive switching instants $t_{i, j+1}>t_{i, j}$, by (iv) it follows,

$$
\begin{aligned}
0 & =\lim _{j \rightarrow \infty} \bar{V}_{i}\left(x_{t_{i, j+1}}\right)-\lim _{j \rightarrow \infty} \bar{V}_{i}\left(x_{t_{i, j}}\right) \\
& \leq \lim _{j \rightarrow \infty}\left[-h_{i}\left(\left\|x_{t_{i, j}}\right\|\right)\right] \leq 0 .
\end{aligned}
$$

It implies $\lim _{j \rightarrow \infty}\left\|x_{t_{i, j}}\right\|=0$ for each $i$, and thus leads to $\lim _{t \rightarrow \infty} x(t+\theta)=0$ for $\theta \in\left[-\tau_{i}, 0\right]$.

\footnotetext{
${ }^{1}$ For the sound definition of the initial condition for subsystem $2, t_{1}>t_{0}+\max _{\{1,2\}}\left(\tau_{i}\right)-\tau_{1}$ is required.
}

Remark 1: If $h_{i}(s) \geq 0$ for $s>0$ in (iv), then the switched time delay system is stable.

Remark 2: Without the restriction to the time interval of activity $t \in\left[t_{j}, t_{j+1}\right)$, conditions (i)-(iii) represent the classic Razumikhin theorem ensuring global uniform asymptotic stability [12] for subsystem $i$ without switching. They guarantee the measure $\bar{V}_{i}\left(x_{t}\right)$ of the states to decrease during the time subsystem $i$ is active. Condition (iv) ensures the sequence of the state measure $\bar{V}_{i}$ to decrease at consecutive switching instants for each subsystem similar to (5). Accordingly, in case of a time delay system without switching, i.e. $\sigma(t)=$ const., Theorem 1 reduces to the original Razumikhin theorem [12] (condition (iv) then follows from (iii) and is no longer needed). For zero time delay, and replacing conditions (i)(iii) by standard Lyapunov stability conditions, the result from [7] on the stability of switched systems with piecewise continuous Lyapunov functions is recovered. For constant time delay, i.e. $\tau_{\sigma(t)}=$ const., a similar result as in [19] is achieved.

\section{B. Common Lyapunov Function for Symmetric Systems}

In this subsection, we restrict ourself to the class of LTI switched time delay systems (2) with symmetric system matrices $A_{i 0}=A_{i 0}^{T}$ and $A_{i 1}=A_{i 1}^{T}$ for all $i \in \mathcal{I}$.

Proposition 1: The system (2) with symmetric system matrices is asymptotically stable for arbitrary ${ }^{2}$ switching sequences if for each subsystem $i$ there exist a real symmetric matrix $P_{i}>0$ and real scalars $\alpha_{i 0}>0, \alpha_{i 1}>0$ such that

$$
\left(\begin{array}{ccc}
M_{i, 0} & -P_{i} A_{i 1} A_{i 0} & -P_{i} A_{i 1}^{2} \\
-A_{i 0}^{T} A_{i 1}^{T} P_{i} & -\alpha_{i 0} P_{i} & 0 \\
\left(A_{i 1}^{2}\right)^{T} P_{i} & 0 & -\alpha_{i 1} P_{i}
\end{array}\right)<0,
$$

where $M_{i, 0}=\frac{1}{\tau_{i}}\left[P_{i}\left(A_{i 0}+A_{i 1}\right)+\left(A_{i 0}+A_{i 1}\right)^{T} P_{i}\right]+\left(\alpha_{i 0}+\right.$ $\left.\alpha_{i 1}\right) P_{i}$.

Sketch Proof: The LMI (6) ensures the asymptotic stability of each subsystem $i$ using the delay-dependent Razumikhin approach and the Lyapunov function $V(x)=x^{T} P_{i} x$. Since $P_{i}>0$, symmetric, there exists a $U_{i}^{-1}=U_{i}^{T}$ such that

$$
U_{i}^{T} P_{i} U_{i}=\Sigma_{i, 0}=\operatorname{diag}\left\{\chi_{i, 1}, \ldots, \chi_{i, n}\right\}
$$

where $\chi_{i, j}>0, j=1, \ldots, n$. Pre- and post-multiply (6) by $U_{i}^{T}$ and $U_{i}$, we have

$$
\left(\begin{array}{ccc}
\bar{M}_{i, 0} & \Sigma_{i, 0} \bar{A}_{i 1} \bar{A}_{i 0} & \Sigma_{i, 0} \bar{A}_{i 1}^{2} \\
-\bar{A}_{i 0}^{T} \bar{A}_{i 1}^{T} \Sigma_{i, 0} & -\alpha_{i 0} \Sigma_{i, 0} & 0 \\
\left(\bar{A}_{i 1}^{2}\right)^{T} \Sigma_{i, 0} & 0 & -\alpha_{i 1} \Sigma_{i, 0}
\end{array}\right)<0,
$$

where $\bar{A}_{i 0}=U_{i}^{T} A_{i 0} U_{i}, \quad \bar{A}_{i 1}=U_{i}^{T} A_{i 1} U_{i}, \bar{M}_{i, 0}=$ $\frac{1}{\tau_{i}}\left[\Sigma_{i, 0}\left(\bar{A}_{i 0}+\bar{A}_{i 1}\right)+\left(\bar{A}_{i 0}+\bar{A}_{i 1}\right)^{T} \Sigma_{i, 0}\right]+\left(\alpha_{i 0}+\alpha_{i 1}\right) \Sigma_{i, 0}$. Pre- and post-multiply (7) by $\Sigma_{i, 0}^{-1}$ and take transpose the resulting matrix, it leads to

$$
\left(\begin{array}{ccc}
\bar{M}_{i, 0}^{\prime} & \Sigma_{i, 0}^{-1} \bar{A}_{i 1} \bar{A}_{i 0} & \Sigma_{i, 0}^{-1} \bar{A}_{i 1}^{2} \\
-\bar{A}_{i 0}^{T} \bar{A}_{i 1}^{T} \Sigma_{i, 0}^{-1} & -\alpha_{i 0} \Sigma_{i, 0}^{-1} & 0 \\
\left(\bar{A}_{i 1}^{2}\right)^{T} \Sigma_{i, 0}^{-1} & 0 & -\alpha_{i 1} \Sigma_{i, 0}^{-1}
\end{array}\right)<0,
$$

${ }^{2}$ By setting $\phi=x\left(t_{0}+\theta\right), \theta \in\left[-\max _{i}\left\{\tau_{i}\right\}, 0\right]$ the initial condition is well-defined for arbitrary switching. 
where $\bar{M}_{i, 0}^{\prime}=\frac{1}{\tau_{i}}\left[\Sigma_{i, 0}^{-1}\left(\bar{A}_{i 0}+\bar{A}_{i 1}\right)+\left(\bar{A}_{i 0}+\bar{A}_{i 1}\right)^{T} \Sigma_{i, 0}^{-1}\right]+$ $\left(\alpha_{i 0}+\alpha_{i 1}\right) \Sigma_{i, 0}^{-1}$. Using $0<\lambda_{i, 1}<1$ which satisfies

$$
\lambda_{i, 1} \chi_{i, 1}+\left(1-\lambda_{i, 1}\right) \chi_{i, 1}^{-1}=1
$$

to $\lambda_{i, 1} \times(7)+\left(1-\lambda_{i, 1}\right) \times(8)$, and using the symmetry of $A_{i 0}$ and $A_{i 1}$, we obtain

$$
\left(\begin{array}{ccc}
\bar{M}_{i, 1} & \Sigma_{i, 1} \bar{A}_{i 1} \bar{A}_{i 0} & \Sigma_{i, 1} \bar{A}_{i 1}^{2} \\
-\bar{A}_{i 0}^{T} \bar{A}_{i 1}^{T} \Sigma_{i, 1} & -\alpha_{i 0} \Sigma_{i, 1} & 0 \\
\left(\bar{A}_{i 1}^{2}\right)^{T} \Sigma_{i, 1} & 0 & -\alpha_{i 1} \Sigma_{i, 1}
\end{array}\right)<0,
$$

where $\Sigma_{i, 1}=\operatorname{diag}\left\{\lambda_{i, 1} \chi_{i, 1}+\left(1-\lambda_{i, 1}\right) \chi_{i, 1}^{-1}, \ldots, \lambda_{i, 1} \chi_{i, n}+\right.$ $\left.\left(1-\lambda_{i, 1}\right) \chi_{i, n}^{-1}\right\}=\operatorname{diag}\left\{1, \bar{\chi}_{i, 2}, \ldots, \bar{\chi}_{i, n}\right\}>0$ and $\bar{M}_{i, 1}=$ $\frac{1}{\tau_{i}}\left[\Sigma_{i, 1}\left(\bar{A}_{i 0}+\bar{A}_{i 1}\right)+\left(\bar{A}_{i 0}+\bar{A}_{i 1}\right)^{T} \Sigma_{i, 1}\right]+\left(\alpha_{i 0}+\alpha_{i 1}\right) \Sigma_{i, 1}$. In the same way for $0<\lambda_{i, 2}<1$, we can derive $\Sigma_{i, 2}=\operatorname{diag}\left\{1,1, \bar{\chi}_{i, 2}, \ldots, \bar{\chi}_{i, n}\right\}>0$. Repeating the procedure, we obtain $\Sigma_{i, n}=I$ and (6) becomes

$$
\left(\begin{array}{ccc}
\frac{2}{\tau_{i}}\left(\bar{A}_{i 0}+\bar{A}_{i 1}\right)+\left(\alpha_{i 0}+\alpha_{i 1}\right) I & -\bar{A}_{i 1} \bar{A}_{i 0} & \bar{A}_{i 1}^{2} \\
-\bar{A}_{i 0}^{T} \bar{A}_{i 1}^{T} & -\alpha_{i 0} I & 0 \\
\left(\bar{A}_{i 1}^{2}\right)^{T} & 0 & -\alpha_{i 1} I
\end{array}\right)<0,
$$

Pre- and post-multiply (III-B) by $U_{i}$ and $U_{i}^{T}$, we have

$$
\left(\begin{array}{ccc}
\frac{2}{\tau_{i}}\left(A_{i 0}+A_{i 1}\right)+\left(\alpha_{i 0}+\alpha_{i 1}\right) I & -A_{i 1} A_{i 0} & A_{i 1}^{2} \\
-A_{i 0}^{T} A_{i 1}^{T} & -\alpha_{i 0} I & 0 \\
\left(A_{i 1}^{2}\right)^{T} & 0 & -\alpha_{i 1} I
\end{array}\right)<0,
$$

From comparison with (6) it follows that each subsystem $i$ satisfies (6) with the Lyapunov function $V(x)=x^{T} x$ representing the common Lyapunov function. Note, that due to the switching time delay, the measure $\bar{V}_{i}\left(x_{t_{k}}\right) \neq \bar{V}_{j}\left(x_{t_{k}}\right)$ for $i \neq j$ is discontinuous at the switching instants in general. For instance if the subsystem dynamics switches at $t_{k}$ from $i$ to $j$ with $\tau_{i}>\tau_{j}$, then $\bar{V}_{i}\left(x_{t_{k}}\right) \geq \bar{V}_{j}\left(x_{t_{k}}\right)$, and vice versa. Due to the common Lyapunov function and its continuity, and the time between any two switches larger than zero, condition (iv) of Theorem 1 is satisfied for arbitrary switching sequences. The values $\bar{V}_{i}\left(x_{t_{k}}\right)$ at the switching instants $t_{k}$ form a strictly decreasing sequence for each $i \in \mathcal{I}$. The conditions (i)-(iii) of Theorem 1 are satisfied for each subsystem $i$ by assumption (6). Thus by application of Theorem 1 , for an arbitrary switching sequence $\lim _{t \rightarrow \infty}\left\|x_{t}\right\|=0$.

Remark 3: The symmetric requirement of system matrices in (2) can be generalized to systems with matrices satisfying

$$
T A_{i 0}=A_{i 0}^{T} T, T A_{i 1}=A_{i 1}^{T} T
$$

for some transformation matrix $T>0$ of appropriate dimension.

Remark 4: The result is an extension to [18] where a common Lyapunov function for symmetric LTI systems without time delay is derived, and to [20] where the time delay is assumed constant.

\section{Towards Performance Oriented Control}

In this section, the potential benefits of the switched time delay systems approach with respect to performance are explored in two novel control approaches for NCS. Aiming at improved control performance, in the first approach the controller is selected depending on the current network time delay. In the second approach, the combined control of the plant and the network QoS is considered with the goal of good control performance and efficient use of network resources. Main difference between these approaches in the view of switched time delay systems is the generation of the switching signal $\sigma(t)$ : It is generated from the time delay monitoring in the time delay dependent controller selection approach, and from the optimization of a cost function depending on the system state and the network cost in the QoS control approach.

\section{A. Network Time Delay Dependent Controller Selection}

With the goal of improving control performance the appropriate controller is selected depending on the time delay. The control system consists of the plant, the communication network, a bank of controllers, and a decision maker, as illustrated in Figure 1. The coomunication network introduces a piecewise constant time delay taking values $\tau_{i}, i \in \mathcal{I}$. Each controller $i$ is designed by a delay-dependent approach, e.g. corollary 1 , to stabilize the plant for the associated time delay value $\tau_{i}$. The decision maker monitors the time delay and chooses the appropriate controller from the controller bank.

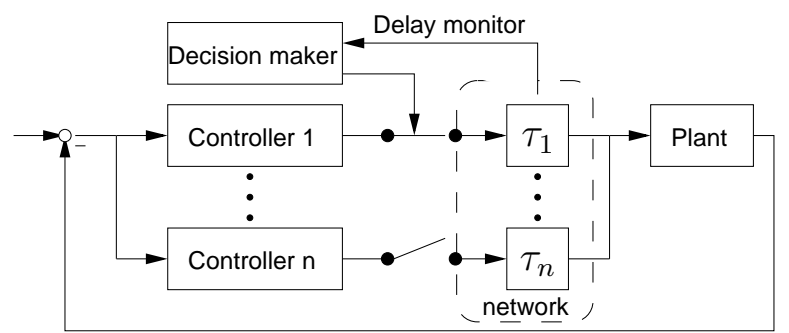

Fig. 1. Control architecture for NCS with network time delay dependent controller selection as a switched time delay system.

In most relevant applications, the time delay in the communication network changes in an unpredictable manner. Accordingly, stability has to be guaranteed for arbitrary switching sequences. For the special class of symmetric system matrices stability is guaranteed by Proposition 1 . The stabilizing controller switching signal can be generated from the time delay monitoring only, i.e. $\sigma(t)=\sigma\left(\tau_{i}(t)\right)$. The time delay and the controller are assumed to switch synchronously.

The control performance benefit is studied in the following simulation example with symmetric system matrices.

Example 1: Consider a communication network where the time delay switches between two possible values, the high time delay $\tau_{1}=250 \mathrm{~ms}$, and the low time delay $\tau_{2}=100 \mathrm{~ms}$. The switched time delay system (2) is given with symmetric system matrices by

$$
A_{10}=A_{20}=\left[\begin{array}{cc}
-1 & -1 \\
-1 & 0
\end{array}\right], A_{i 1}=k_{i}\left[\begin{array}{cc}
-1 & 0 \\
0 & -1
\end{array}\right]
$$

where $A_{10}=A_{10}^{T}=A_{20}=A_{20}^{T}$ is the unstable second order plant, $A_{i 1}$ represents the state feedback gain matrix with a pre-defined symmetric structure for simplicity. The 
gains $k_{i}$ are determined by iteratively solving (4) with $\alpha_{10}=\alpha_{11}=1, \alpha_{20}=\alpha_{21}=2$ using the MATLAB LMI toolbox. The gains $k_{1}=1.79$ and $k_{2}=3.01$ represent the highest gains where the LMI (4) is still feasible. The corresponding positive definite matrices are

$P_{1}=\left[\begin{array}{cc}1.757 & -0.6888 \\ -0.6888 & 2.446\end{array}\right], P_{2}=\left[\begin{array}{cc}0.6258 & -0.1375 \\ -0.1375 & 0.7652\end{array}\right]$.

With the controller gain $k_{1}$ designed for the high time delay $\tau_{1}$, the system is stabilized also for low time delay $\tau_{2}$, a feasible solution of (4) exists with

$$
P_{*}=\left[\begin{array}{cc}
0.2776 & -0.1788 \\
-0.1788 & 0.4564
\end{array}\right] \text {. }
$$

According to Proposition 1 the system is stable for arbitrarily switching time delay, for both cases, with the constant controller $k_{1}$ designed for the worst case (highest) time delay, and with synchronously switching controller. In simulations the control performance for these two cases is compared. The network initially provides a low time delay communication $\tau_{2}$. The subsequent change of the time delay is depicted in Figure 2(a). The initial condition for the state is given by $x_{0}^{T}=[0,1],-\tau_{2}<\theta<0$. The system trajectories are computed using the dde 3 solver from MATLAB. Without adaption of the controller to a change in network time delay, i.e. the worst case controller $k_{1}$, the state trajectories show a rather slow convergence towards zero, see dashed line in Figure 2(b),(c). The trajectory stays within a ball around the origin of radius $\|x\|=0.1$ after $t_{0.1}=1.15 \mathrm{~s}$. With synchronously switching controller the state trajectories converge faster to the origin, with $t_{0.1}=0.59 \mathrm{~s}$. Clearly, there is performance benefit from using the controller switching approach with piecewise constant time delays.

Remark 5: For the general class of non-symmetric systems according to Theorem 1 the decision maker needs to monitor the evolution of the Lyapunov functions of the subsystems additionally to the time delay. The switching signal has to be generated as a function of the time delay and the Lyapunov function value $\sigma(t)=\sigma\left(\tau_{i}(t), \bar{V}_{i}(t)\right)$. If all subsystems are asymptotically stable then only condition (iv) of Theorem 1 has to be evaluated, see Remark 2. There may, however, exist time delay switching instants where the evolution of the Lyapunov function does not allow the switching of the controller. How the stability of the system can still be guaranteed is subject of future research. For nonsymmetric systems, it is furthermore desirable to replace the Lyapunov function condition by a condition which is easier to check, e.g. by a dwell-time condition.

Remark 6: Note that in the considered system (2), controller an plant are lumped into a single delay differential functional assuming no state jumps, and the initial condition $x_{t}$ for each time delay subsystem to be well defined after switchings. However, this assumption is no longer true in a real NCS application due to the spatial separation of controller and plant as the following shows. Consider the plant $\dot{x}=f\left(x, u\left(t-\tau_{i}\right)\right)$ and the proportional state feedback controller $u(t)=K_{i} x(t)$. Controller and time delay are

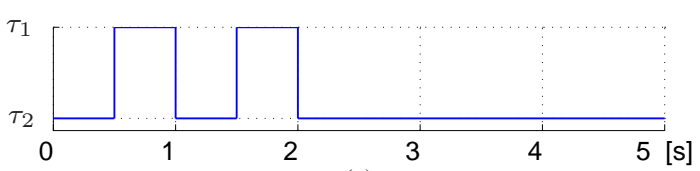

(a)
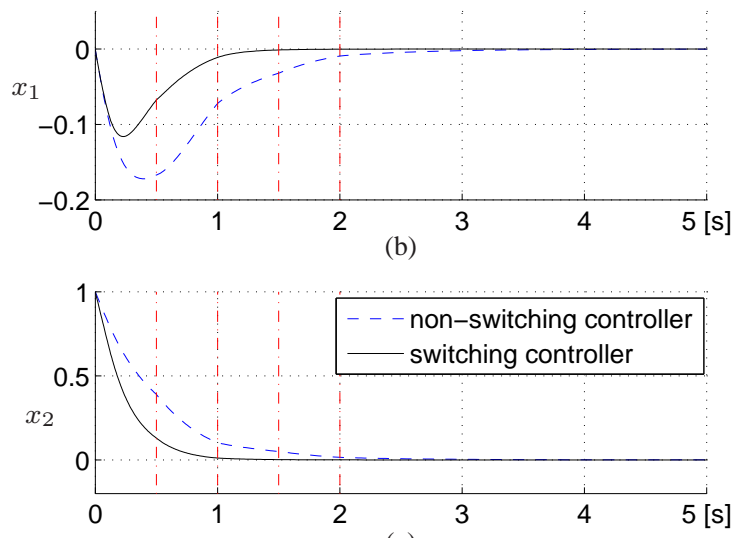

(c)

Fig. 2. Time delay evolution (a) and state trajectories with time delay dependent controller selection (solid) and without, using the worst case controller $k_{1}$ designed for time delay $\tau_{1}$ (dashed).

switched at time instant $t_{q}$ to $K_{j}$ and $\tau_{j}$, respectively. If $\tau_{i}>\tau_{j}$, then there is an overlap in the control signal $u$ arriving delayed at the plant over a time interval $\tau_{i}-\tau_{j}$. Accordingly, if $\tau_{i}<\tau_{j}$, the control signal remains undefined for the time interval $\tau_{j}-\tau_{i}$. The definition of the control signal over this time interval represents a degree of freedom during the design. It has an influence on the evolution of the Lyapunov function. A deeper analysis of this case is beyond the scope of this paper.

Remark 7: Markovian jump systems (MJS) with modedependent time delays [21] represent an alternative approach for the controller switching depending on piecewise constant time delay. Main difference is that the design of such control system relies on the knowledge of the probabilistic transition rates of the time delay values, while in the proposed approach the time delay values are monitored. A comparison of both approaches are subject to further research.

\section{B. Quality-of-Service Control}

Quality-of-Service (QoS) refers to the capability of a network to provide different communication quality to different network traffic. In fact, it allows the adjustment, hence the control of the communication quality. In the current IPv6 a finite number of data classes is already implemented. Guaranteed high communication quality, e.g. low time delay, requires the provision of large network resources, i.e. induces high network cost. Considering the finite resources of a network it is desirable that every application only consumes as much of them as required to guarantee a certain level of performance. Inspired by the QoS concept a novel control concept is proposed here aiming at the conjoint control of the control system itself and indirectly of the the network resources. 


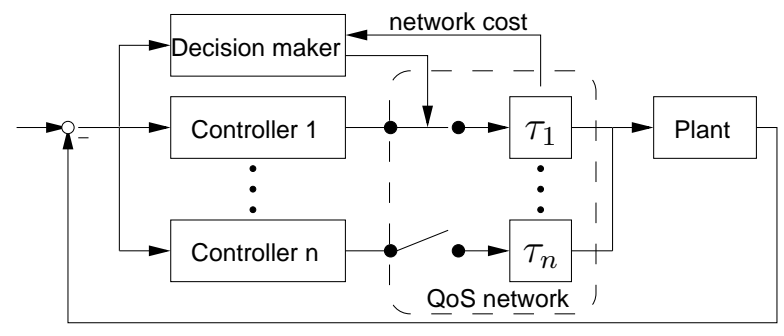

Fig. 3. Control architecture for NCS with QoS control as a switched time delay system.

The basic control architecture is similar to the previous application as illustrated in Fig.3. The finite number of possible time delay values is assumed to be known. For each time delay value $\tau_{i}$ a stabilizing controller $i$ is designed using a delay-dependent approach. The decision maker chooses the appropriate time delay-controller pair. Main difference to the delay-dependent controller selection approach is the generation of the switching signal, i.e. the switching logic in the decision maker.

The goal here is to balance control performance versus network cost. For good control performance, generally, a low time delay connection is desirable, however high network cost are induced then. The cost-performance trade-off can be formulated as an optimization problem with the goal to find the optimal switching signal $\sigma(t)$. As control performance measure here the time integral over a quadratic function of the states is considered. The network cost are assumed to be time-based, i.e. increase with connection usage time. The resulting optimization problem can be stated as follows

$$
\min _{\sigma(t)} J=\int_{0}^{\infty} x^{T}(t) Q(t) x(t)+\eta_{\sigma(t)} \mathrm{d} t
$$

where $Q(t)$ is a weighting matrix of appropriate dimension and $\eta_{\sigma(t)}=\eta\left(\tau_{\sigma(t)}\right)$ represents the network cost associated with a certain time delay. The solution to this optimization problem is generally difficult to find as it is hybrid, nonconvex in general and contains delay differential equations. It is beyond the scope of this paper to provide a deeper analysis. Hybrid optimal control for systems without time delay is investigated e.g. [16], [22]. In this work the two-stageprocedure proposed in [16] is adapted to solve the hybrid optimal control problem with piecewise constant time delay numerically over a finite horizon. Number $k$ and sequence of switchings $\left\{\left(t_{1}, \sigma\left(t_{1}\right)\right), \cdots,\left(t_{k}, \sigma\left(t_{k}\right)\right)\right\}$ are fixed a priori, in the second stage the switching times are optimized. An alternative hybrid optimal control approach is proposed in [22], however, without considering time delay. For symmetric systems Proposition 1 applies straightforwardly, i.e. the solution $\sigma^{*}(t)$ of the optimization problem stabilizes the switched time delay system and guarantees a balanced trade-off between control performance and network cost. For non-symmetric systems additionally the condition (iv) of Theorem 1 has to be satisfied for stability, conditions (i)-(iii) are satisfied by the stability of each subsystem, see Remark 2. Accordingly, the optimization problem (9) has to be solved subject to the constraint (iv) of Theorem 1. The resulting solution $\sigma^{*}(t)$ stabilizes the switched time delay system, and achieves the desired trade-off.

The benefits of QoS control are studied in the following simulation example with non-symmetric system matrices. A comparison between the QoS control approach and nonswitching approaches is performed with respect to control performance and network cost.

Example 2: Consider a QoS communication network with two different traffic classes, with a time delay of $\tau_{1}=250 \mathrm{~ms}$ and $\tau_{2}=100 \mathrm{~ms}$, respectively. The corresponding network cost are given by $\eta\left(\tau_{1}\right)=1 \mathrm{~s}^{-1}$ and $\eta\left(\tau_{2}\right)=3 \mathrm{~s}^{-1}$, i.e. higher cost for lower time delay. An unstable unsymmetric plant is considered, the controller structure is pre-defined as in Example 1

$$
A_{10}=A_{20}=\left[\begin{array}{cc}
1 & -1 \\
1 & 0
\end{array}\right], A_{i 1}=k_{i}\left[\begin{array}{cc}
-1 & 0 \\
0 & -1
\end{array}\right]
$$

with $A_{10}=A_{20}$ the plant in (2), and $A_{i 1}$ the controllers. The feedback gains $k_{i}$ are designed in the same way as in Example 1, i.e. by determining the largest value where the LMI (4) is still feasible with $\alpha_{10}=\alpha_{20}=1$ and $\alpha_{11}=\alpha_{21}=2$ through iteratively solving (4). The result is computed to $k_{1}=1.58, k_{2}=2.93$ with the corresponding positive definite matrices

$$
P_{1}=\left[\begin{array}{cc}
24.35 & -12.18 \\
-12.18 & 24.35
\end{array}\right], P_{2}=\left[\begin{array}{cc}
10.53 & -5.281 \\
-5.281 & 10.53
\end{array}\right] .
$$

The optimization problem (9) is solved numerically with $Q(t)=I$. Following the approach in [16] the number of switches $k=2$ is fixed prior to optimization. The initial mode is defined to be $\sigma(0)=1$, i.e. initially controller $k_{1}$ with time delay $\tau_{1}$ is active. The is order of the switchings is fixed as $\left.\left\{\left(t_{1}, 2\right),\left(t_{2}, 1\right)\right)\right\}$, meaning that switching into subsystem 2 takes place at $t=t_{1}$. The initial condition
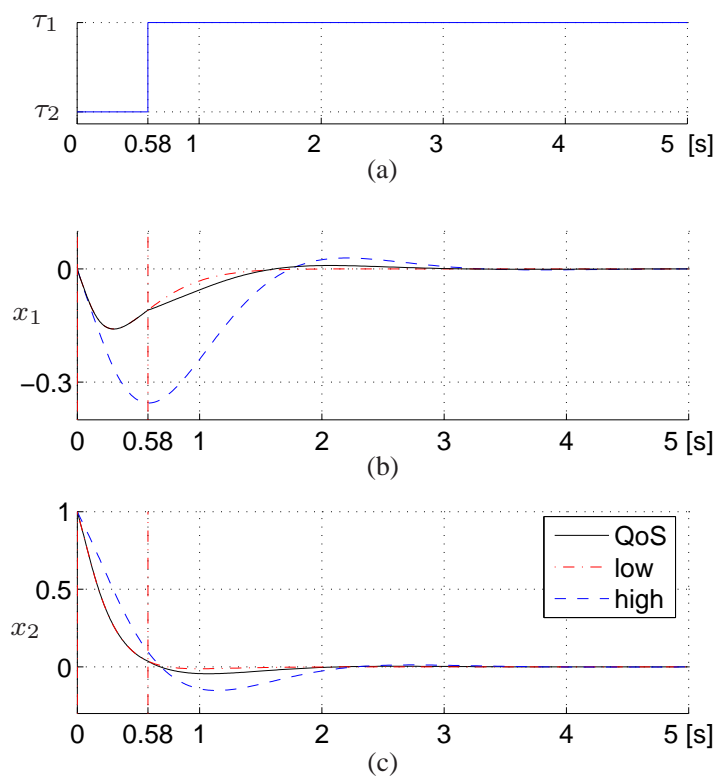

Fig. 4. Time delay evolution (a) and state trajectories for QoS controlled NCS (solid), i.e. the switched time delay system; for comparison the state trajectories for controller $k_{1}$ with high time delay $\tau_{1}$ (dashed), and controller $k_{2}$ with low time delay $\tau_{2}$ (dash-dotted). 
TABLE I

CONTROL PERFORMANCE AND INDUCED NETWORK COST.

\begin{tabular}{|l|c|c|}
\hline & $t_{0.1}[\mathrm{~s}]$ & Network cost [units] \\
\hline \hline QoS control & 0.66 & 6.15 \\
controller $1 / \tau_{1}$ only & 1.58 & 5 \\
controller $2 / \tau_{2}$ only & 0.63 & 15 \\
\hline
\end{tabular}

for the state is given by $x_{0}^{T}=[0,1],-\tau_{1}<\theta<0$. The optimization problem is solved off-line for a time horizon of $5 \mathrm{~s}$ using the fminsearch algorithm from the MATLAB optimization toolbox and the dde 23 solver resulting in the switching times $t_{1}=0 \mathrm{~s}$ and $t_{2}=0.58 \mathrm{~s}$, respectively. The first switch is degenerated, it redefines the initial mode to controller $2 / \tau_{2}$. Hence there is only one switch between the subsystems, the overall switched system is stable due to the stability of the subsystems. In Figure 4 the evolution of the time delay and the state trajectories are presented for the QoS controlled system, as well as for the standard approach with high or low time delay only, i.e. without switching. The trajectories of the QoS controlled system stay within the ball of radius $\|x\|=0.1$ after $t_{0.1}=0.66 \mathrm{~s}$, close to the unswitched system with low delay communication $(+4.6 \%)$, see Table I. However, with QoS control the low delay connection is active for $0.58 \mathrm{~s}$ only, the network cost are $60 \%$ less than for the unswitched system with low delay. As desired, a trade-off between control performance and network cost is achieved.

Open problems that will be addressed in future research includes the following: The optimization of the switching instants is currently performed off-line. An implementable online optimization algorithm that computes the next switching instants under the stability constraint (iv) of Theorem 1is desirable.

In summary, the proposed delay-dependent controller selection approach as well as the QoS control concept seem very promising for future NCS applications.

\section{CONCLUSION}

Motivated by novel control approaches for networked control systems (NCS) this paper presents a first approach towards switched time delay systems with piecewise constant time delay and gives sufficient stability conditions. For a switched system with time delay subsystems a piecewise Lyapunov function is constructed using the Razumikhin approach. Asymptotic stability is guaranteed by a strictly decreasing sequence of the values of a measure for the retarded states. For the restricted class of symmetric systems a common Lyapunov function is derived ensuring asymptotic stability for arbitrary switching sequences. The benefits of the switched time delay system approach for the performance is demonstrated in two novel control approaches for NCS. In the first approach the time delay is monitored and an appropriate controller is selected. The second approach targets the conjoint control of system controller and network QoS, i.e. time delay, such that an optimal trade-off between network resource usage and control performance is achieved. Both approaches are very promising for future NCS.

\section{ACKNOWLEDGMENTS}

This work was supported in part by the Japanese Society for the Promotion of Science (JSPS) by a Postdoctoral Fellowship for Foreign Researchers granted to the first author. The helpful comments of the anonymous reviewers are appreciated.

\section{REFERENCES}

[1] W. Zhang, M. S. Branicky, and S. M. Philips, "Stability of network control systems," IEEE Control Systems Magazine, vol. 21, pp. 84-99, February 2001.

[2] Y. Tipsuwan and M. Y. Chow, "Control methodologies in network control systems," Control Engineering Practice, vol. 11, pp. 10991011, 2003

[3] S. Petersson, Analysis and Design of Hybrid Systems. PhD thesis, Chalmers University of Technology, Göteborg, Sweden, 1999.

[4] D. Liberzon and A. S. Morse, "Basic problems in stability and design of switched systems," IEEE Control Systems Magazine, vol. 19 pp. 59-70, October 1999.

[5] P. Peleties and R. DeCarlo, "Asymptotic stability of m-switched systems using lyapunov functions," in Proceedings of the $31^{\text {th }}$ Conference on Decision and Control, (Tucson, Artzons), pp. 3438-3489, 1992.

[6] R. A. Decarlo, M. S. Branicky, S. Petersson, and B. Lennartson, "Perspectives and results on the stability and stabilization of hybrid systems," in Proceeding of the IEEE, vol. 88.

[7] H. Ye, N. Michel, and L. Hou, "Stability theory for hybrid dynamical systems," IEEE Transactions on Automatics Control, vol. 43, pp. 461474, April 1998.

[8] A. S. Morse, "Supervisory control of families of linear set-point controllers - part 1: exact matching," IEEE Transactions on Automatic Control, vol. 41, pp. 1413-1431, October 1996.

[9] J. Hespanha and A. Morse, "Stability of switched systems with average dwell-time." Technical report, EE-Systems, University of Southern California/ Lab. for Control Science and Eng., Yale University, 1999.

[10] J.-P. Richard, "Time-delay systems: an overview of some recent advances and open problems," automatica, vol. 39, pp. 1667-1694, 2003.

[11] J. K. Hale and S. M. V. Lunel, Introduction to Functional Differential Equations. Springer-Verlag, 1993.

[12] K. Gu, V. L. Kharitonov, and J. Chen, Stability of Time-Delay Systems. Birkhäuser, 2003.

[13] Q.-Y. Tong, G.-F. Yan, and G.-Z. Zhao, "Stability analysis of hybrid systems with time-varing delayed perturbation via single lyapunov function," in Proceedings of the Second International Conference on Machine Learning and Cybernetics, pp. 919-922, 2003.

[14] G. Zheng, M. Tan, and Y. Song, "An approach to analyze the stability of a class of hybrid systems wiht delay," in Proceeding of $5^{\text {th }}$ World Congress on Intelligent Contorl and Automation, (Hangzhou, P.R. China).

[15] P. Yan and H. Özbay, "Stability analysis of switched time-delay systems," in Proceedings of the 16th IFAC World Congress, (Prague, Czech Republic), 2005

[16] X. Xu and P. J. Antsaklis, "An approach for solving general switched linear quadratic optimal control problems," in Proceedings of the $40^{\text {th }}$ Conference on Decision and Control, (Orlando, Florida USA), pp. 2478-2483, 2001

[17] D. Liberzon, Switching in Systems and Control. Birkhäuser, 2003.

[18] K. S. Narendra and J. Balakrishnan, "A Common Lyapunov Function for Stable LTI Systems with Commuting A-Matrices," IEEE Transactions on Automatic Control, vol. 39, pp. 2469-2471, December 1994

[19] R. Yuan, Z. Jing, and L. Chen, "Uniform asymptotic stability of hybrid dynamical systems with delay," IEEE Transactions on Automatic Control, vol. 48, pp. 344-348, February 2003.

[20] G. Zhai, Y. Sun, X. Chen, and A. Michel, "Stability and L2 Gain Analysis for Switched Symmetric Systems with Time Delay," in Proceedings of the American Control Conference, (Denver, Colorado, US), pp. 2682-2687, 2003

[21] E.-K. Boukas and Z.-K. Liu, Deterministic and Stochastic Time Delay Systems. Birkhäuser, 2002.

[22] S. C. Bengea and R. A. DeCarlo, "Optimal Control of Switching Systems," automatica, vol. 41, pp. 11-27, 2005. 